\title{
DESEMPENHO DE FORANSULFURON + IODOSULFURON E IMAZAPIC + IMAZAPYR EM BIÓTIPOS DE Bidens spp.
}

\author{
Nunes, A.L. ${ }^{1}$; Vidal, R.A. ${ }^{2}$; Trezzi, M.M. ${ }^{3}$ \\ ${ }^{1}$ Eng. Agr., mestrando do Programa de Pós-Graduação em Fitotecnia da Universidade \\ Federal do Rio Grande do Sul (UFRGS), Caixa Postal 15.100, CEP 90.000-970, Porto \\ Alegre, RS; \\ ${ }^{2}$ Eng. Agr., Ph.D., Professor da UFRGS, bolsista do CNPq, e.mail: \\ ribas.vidal@pesquisador.cnpq.br; \\ ${ }^{3}$ Eng. Agr., Dr., Prof. do CEFET-Unidade de Pato Branco, PR.
}

\section{RESUMO}

Bidens spp. encontra-se presente em $74 \%$ das lavouras do planalto central do Rio Grande do Sul. A espécie Bidens pilosa foi a primeira planta daninha a ser relatada como resistente aos herbicidas inibidores da enzima acetolactato sintase (ALS) no Brasil. Foransulfuron + iodosulfuron e imazapic + imazapyr são herbicidas inibidores da enzima ALS usados nacultura de milho e não há relatos na literatura sobre a ação destes herbicidas em biótipos resistentes de Bidens spp. O objetivo deste trabalho foi avaliar o desempenho de foransulfuron + iodosulfuron e imazapic + imazapyr em biótipos de Bidens ssp. oriundas de vários locais do país. Foram instalados experimentos em casa de vegetação na Faculdade de Agronomia da UFRGS. Sementes de Bidens spp. foram semeadas em bandejas com capacidade para $2000 \mathrm{ml}$, com substrato de solo-areia (2:1). Após emergência, plântulas foram transplantadas para vasos de $300 \mathrm{ml}$ e mantidas sem déficit hídrico. Quando as plântulas atingiram quatro folhas verdadeiras foram aspergidas com os herbicidas em até 8 vezes a dose recomendada, em quatro repetições. Aos 21 dias após a aplicação, avaliou-se o controle das plantas. Os resultados foram submetidos à análise de variância e quando constatada interação entre biótipo e dose ajustaram-se curvas de resposta à dose, para cada biótipo. Os resultados confirmaram diferença de sensibilidade aos herbicidas entre os biótipos testados. Os biótipos suscetíveis a outros inibidores de ALS, confirmaram a sua susceptibilidade aos herbicidas foransulfuron + iodosulfuron e imazapic + imazapyr. Mas, os biótipos resistentes a outros inibidores de ALS também confirmaram sua resistência a estes herbicidas. Conclui-se que foransulfuron + iodosulfuron e imazapic + imazapyr são novos herbicidas para a cultura de milho e são iguamente ineficientes sobre os biótipos de Bidens spp resistentes aos inibidores da ALS.

Palavras-chave: herbicida, resistência, rotação de cultura, picão-preto 


\title{
Performance of foransulfuron + iodosulfuron and imazapic + imazapyr on Bidens spp. biotypes
}

\begin{abstract}
Bidens spp. especies are present in $74 \%$ of the farms of central plateaus of the state of Rio Grande Do Sul, Brazil. The species Bidens pilosa was the first weed to be documented as resistant to herbicides inhibitors of the enzyme acetholactate sinthase (ALS), in Brazil. Foransulfuron + iodosulfuron and imazapic + imazapyr are ALS inhibitors recently launched to use on the corn crop. Since, there is no record of the action of these herbicidas on resistant Bidens spp. biotypes, the objective of this work was to evaluate the performance of foransulfuron + iodosulfuron and imazapic + imazapyr on biotypes collected in several places in Brazil. Experiments were conducted in the greenhouse at Federal University at Rio Grande do Sul. Seeds of Bidens spp. had been sown in trays with capacity for $2000 \mathrm{ml}$, with soil:sand substratum (2:1). After emergence, seedlings were transfered to vases with $300 \mathrm{ml}$ capacity and kept irrigated. When seedlings had reached the four leaved stage they were sprayed with the herbicides with rates from 0 up to 8 times the recommended dose. The experimental design was a complete random design with four replicates. At 21 days after the application it was evaluated the plant control. The results had been submitted to the analysis of variance and when evidenced interaction of biotype and rate, dose-response curves had been adjusted, for each biotype. The results confirmed difference of sensitivity to the herbicides between the tested biotypes. The biotypes susceptible to other ALS inhibitors had confirmed its susceptibility to the herbicidas foransulfuron + iodosulfuron and imazapic + imazapyr. But, the biotypes resistant to other ALS-inhibitors were also resistant to these herbicidas. The mixtures of foransulfuron + iodosulfuron and imazapic + imazapyr are new herbicidas for the corn crop but do not solve the problem of ALS-resistance in Bidens spp.
\end{abstract}

Key-words: herbicides, resistance, crop rotation, hairy beggarticks.

\section{INTRODUÇÃO}

Em mais de 40 países as espécies Bidens spp. são consideradas como as espécies daninhas mais importantes nas culturas (Kissmann \& Groth, 1992). No Planalto Central do Rio Grande do Sul as espécies Bidens spp. encontram-se presentes em 74\% das lavouras (Bianchi, 1996). Em 1994, a espécie Bidens pilosa foi a primeira planta daninha a ser relatada como resistente aos herbicidas no Brasil (Heap, 2005), encontrando-se amplamente distribuídas em todas as regiões.

A extensão de áreas agrícolas detectadas com presença de biótipos resistentes de plantas daninhas no Brasil é superior a 2 milhões de hectares e está aumentando a uma taxa elevada, com várias espécies já diagnosticadas com este problema, principalmente em lavouras de soja e arroz. 
O controle químico de Bidens spp. é realizado predominantemente por herbicidas inibidores da enzima ALS. Estes produtos são muito utilizados na agricultura devido à alta eficácia no controle de várias espécies de plantas daninhas, às baixas doses recomendadas, a baixa toxicidade aos mamíferos e a alta seletividade para as culturas. Na atualidade, a ocorrência de populações de plantas daninhas resistentes aos herbicidas inibidores de ALS tem aumentado gradativamente devido ao seu uso repetido durante vários anos (Powles \& Holtum, 1994). No Brasil os inibidores de ALS compõem uma das classes mais numerosas de herbicidas (Trezzi \& Vidal, 2001).

O principal mecanismo que confere resistência aos inibidores de ALS para biótipos no mundo é a alteração no local de ação do herbicida, fazendo com que estes produtos não tenham atividade herbicida. A falta de sensibilidade da enzima aos herbicidas que atuam inibindo sua atividade é a causa da resistência de biótipos de Bidens pilosa L. aos inibidores de ALS. Isto ocorre devido a alteração em sua estrutura, resultando da substituição de certos aminoácidos na enzima (Christoffoleti, 2004).

Foransulfuron + iodosulfuron e imazapic + imazapyr são inibidores da enzima ALS recentemente registrados para a cultura de milho com o nome comercial de Equip plus e Onduty, respectivamente. Não há relatos na literatura sobre a ação destes herbicidas em biótipos resistentes. O objetivo deste trabalho foi avaliar 0 desempenho de foransulfuron + iodosulfuron e imazapic + imazapyr em oito biótipos de Bidens spp. oriundos de vários locais do país.

\section{MATERIAIS E MÉTODOS}

Em casa de vegetação na Faculdade de Agronomia da UFRGS, as sementes de Bidens spp. foram semeadas em bandejas com capacidade para $2000 \mathrm{ml}$, com substrato de solo-areia (2:1). Após emergência, três plântulas foram transplantadas para vasos de $300 \mathrm{ml}$.

Aos 40 dias após a emergência foi realizada a aplicação dos herbicidas, quando as plantas de Bidens spp. apresentavam quatro folhas definitivas. A aplicação foi feita com pulverizador pressurizado com $\mathrm{CO}_{2}$ com pressão de $200 \mathrm{kPa}$, com ponta de pulverização do tipo 110.01, distribuindo a calda uniformemente. Em todos os tratamentos foi utilizada vazão de 100 L.ha ${ }^{-1}$. No momento da aplicação a temperatura ambiente era de $26{ }^{\circ} \mathrm{C}$ e a umidade relativa do ar era de $83 \%$. Os vasos foram irrigados por capilaridade, sendo colocados em bandejas com uma lâmina d'água de $10 \mathrm{~mm}$ de altura.

Foram realizados dois experimentos com delineamento experimental completamente casualizado com arranjo bifatorial de 4 repetições. No primeiro experimento, foi aplicada a associação dos herbicidas foransulfuron + iodosulfuron, onde o fator A consistiu nos biótipos B1 e B3 (resistentes) e nos biótipos B6 e B8 (suscetíveis) e o fator B consistiu nas doses: 0,$0 ; 25,0 ; 33,3 ; 50,0 ; 66,6 ; 100,0 ; 200,0$; 
400,$0 ; 600,0$ e $800,0 \%$ da dose recomendada pelo fabricante (300 $\mathrm{g}$ i.a ha ${ }^{-1}$ para foransulfuron $+20 \mathrm{~g}$ i.a ha ${ }^{-1}$ para iodosulfuron).

No segundo experimento, foi aplicada a associação dos herbicidas imazapic + imazapyr, onde o fator A consistiu nos biótipos B2 e B5 (resistentes) e nos biótipos B7 e B9 (suscetíveis) e o fator B consistiu nas doses: 0,0; 25,0; 33,3; 50,0; 66,6; 100,$0 ; 200,0 ; 400,0 ; 600,0$ e $800,0 \%$ da dose recomendada pelo fabricante $(52,5$ g.i.a ha $^{-1}$ para imazapic $+17,5 \mathrm{~g}$ i.a ha ${ }^{-1}$ para imazapyr). Em ambos os experimentos, foi adicionada a calda de aplicação surfactante não iônico, na dose de 0,25\%.

Aos vinte e um dias após a aplicação dos herbicidas (DAT), foram realizadas as avaliações visuais de controle das plantas, comparada com a testemunha, sendo atribuído $0 \%$ quando não existia injúria e $100 \%$ quando havia morte das plantas pelos herbicidas.

Os resultados coletados foram submetidos à análise de variância através do teste F. Quando constatadas diferenças significativas, foi realizado o teste de comparação de médias pelo teste $t$ (DMS) ao nível de $5 \%$ de probabilidade do erro experimental. $O$ ajuste das curvas foi realizado pelo programa Origin 5.0 Professional. Foi calculada a dose necessária para o controle de $50 \%$ dos indivíduos de cada biótipo ( $\left(\mathrm{L}_{50}\right)$. $\mathrm{E}$ através desta variável foi calculado o fator de resistência (FR) dividindo o $\mathrm{DL}_{50}$ dos biótipos resistentes pelo $\mathrm{DL}_{50}$ dos biótipos suscetíveis.

\section{RESULTADOS E DISCUSSÕES}

Para foransulfuron + iodosulfuron, o controle na dose 100\%, aos 07 (DAT) foi de $5,2,12$ e 13\% e aos 14 DAT foi de 28, 9, 63 e 73\% para os biótipos B1, B3, B6 e B8, respectivamente. Aos 21 DAT, as curvas de dose-resposta para os biótipos relativos ao foransulfuron + iodosulfuron indicam que o modelo linear se ajustou aos resultados obtidos com os biótipos $\mathrm{B} 3$, B6 e B8, com $\mathrm{R}^{2}$ igual a 0,92, 0,95 e 0,95, respectivamente. Para o biótipo $\mathrm{B} 1$ os resultados apresentados se ajustaram à equação da hipérbole com $\chi^{2}$ igual a 11,5 (Figura 1).

Nos biótipos suscetíveis a outros inibidores de ALS, B6 e B8, com a dose recomendada (100\%) os níveis de controle chegaram a 90 e $100 \%$ respectivamente, confirmando a susceptibilidade aos dois inibidores da ALS testados. Nos biótipos resistentes a outros inibidores de ALS, B1 e B3, os níveis de controle obtidos com a dose de $100 \%$ foram de 27 e 5\%, respectivamente. Quando se utilizou $800 \%$ da dose recomendada a taxa de controle atingiu $69 \%$ para o biótipo B1 e 50\% para o biótipo B3. Estes resultados confirmam a resistência cruzada dos biótipos B1 e B3 aos herbicidas foransulfuron + iodosulfuron.

Para imazapic + imazapyr, o controle na dose 100\%, aos 07 DAT foi de 3, 2, 25 e 70\% e aos 14 DAT foi de 70, 3, 55 e 95\% para os biótipos B2, B5, B7 e B9, respectivamente. Aos 21 DAT, as curvas de dose-resposta para os biótipos relativos ao imazapic + imazapyr indicam que o modelo linear se ajustou aos resultados obtidos com os biótipos B5 e B7, com $\mathrm{R}^{2}$ igual a 0,94 e 0,90, respectivamente. Para os biótipos B2 e B9, os resultados apresentados se ajustaram à equação da hipérbole com $\chi^{2}$ igual a 46,8 e 0,15, respectivamente (Figura 2). 


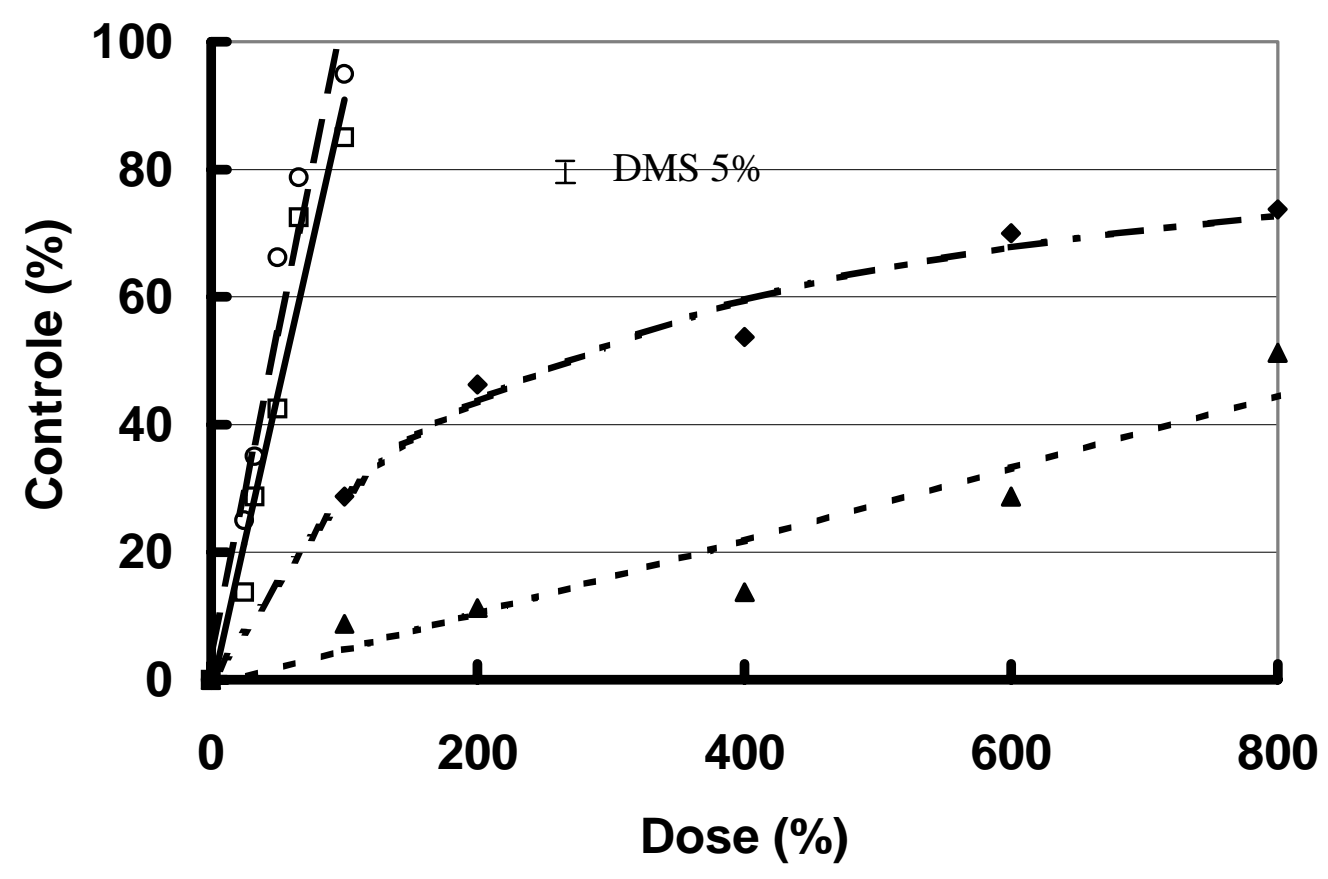

Figura 1 - Controle (\%) do Biótipo B1 (•), Biótipo B3 ( $\mathbf{\Delta}$ ) (Resistentes), Biótipo B6 () e Biótipo B8 (०) (Suscetíveis) de Bidens spp., aos 21 DAA com foransulfuron + iodosulfuron. Linhas indicam as equações ajustadas e pontos representam a média de 4 repetições. Barra de DMS indica a diferença mínima significativa pelo teste $t$, para comparar dois tratamentos quaisquer ao nível de probabilidade indicada.

Nos biótipos suscetíveis a outros inibidores de ALS, B7 e B9, com a dose recomendada (100\%) os níveis de controle chegaram a $94 \%$ e $100 \%$ respectivamente, confirmando sua susceptibilidade aos inibidores da ALS. No biótipo resistente a outros inibidores de ALS, B5, o nível de controle obtido com a dose $100 \%$ foi de $3 \%$. Quando se utilizou $800 \%$ da dose recomendada, o controle foi de $12 \%$. Estes resultados confirmam a resistência do biótipo B5 à associação de imazapic + imazapyr. 


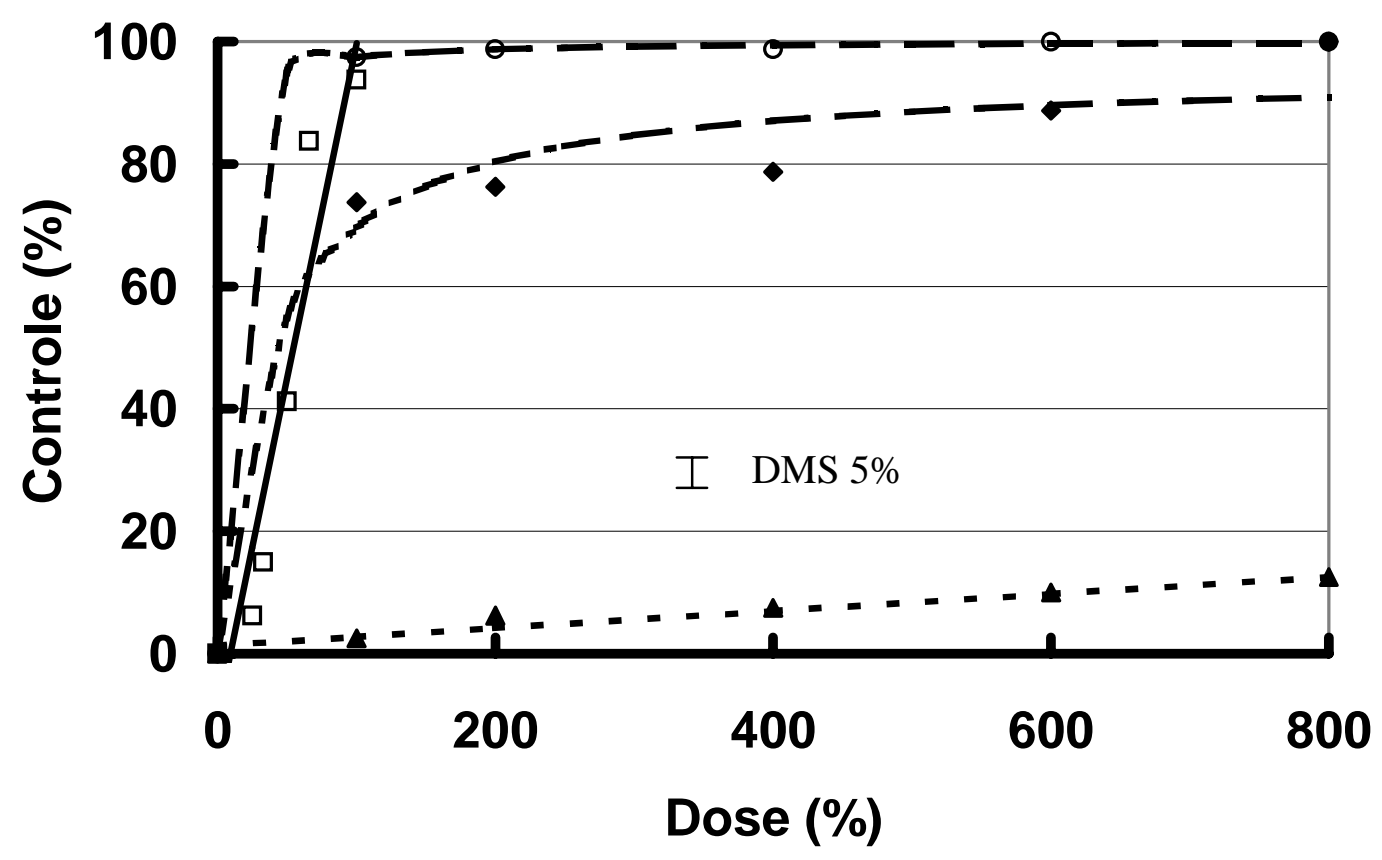

Figura 2 - Controle (\%) do Biótipo B2 (•), Biótipo B5 (ム) (Resistentes), Biótipo B7 () e Biótipo B9 (०) (Suscetíveis) de Bidens spp., aos 21 DAA com imazapic + imazapyr. Linhas indicam as equações ajustadas e pontos representam a média de 4 repetições. Barra de DMS indica a diferença mínima significativa pelo teste $t$, para comparar dois tratamentos quaisquer ao nível de probabilidade indicada.

No biótipo B2, o nível de controle obtido com a dose $100 \%$ foi de $69 \%$. Quando se utilizou $800 \%$ da dose recomendada, o controle foi de $90 \%$. Os menores níveis de controle podem ser resultantes de seleção direcional, dadas as dificuldades de controle do biótipo com os herbicidas inibidores de ALS em relação aos biótipos suscetíveis. O uso continuo de herbicidas inibidores de ALS deixará o biótipo B2 como o biótipo B5, pois mesmo usando $800 \%$ da dose recomendada, $10 \%$ dos indivíduos sobrevivem e se reproduzem, gerando indivíduos resistentes e com o tempo, isso mudará a composição populacional como mecanismo de sobrevivência, diminuindo a sensibilidade aos herbicidas inibidores da ALS.

Os valores de $\mathrm{DL}_{50}$ para os biótipos resistentes foram superiores aos dos biótipos suscetíveis (Tabela 1). Comparando os biótipos resistentes tratados com foransulfuron + iodosulfuron, a $D L_{50}$ para $B 3$ foi 3 vezes superior a $D_{50}$ do $B 1$, caracterizando-se assim, que B3 está em um estágio de resistência mais avançado, para essa associação de herbicidas, do que B1. 
Para os biótipos resistentes tratados com imazapic + imazapyr, a $\mathrm{DL}_{50}$ para B5 foi quase 10 vezes superior a $\mathrm{DL}_{50}$ do B2. Utilizando $800 \%$ da dosagem recomendada não se registrou danos superiores a $50 \%$ aos indivíduos dos biótipos B3 e B5 (Figuras 1 e 2).

Tabela 1 - Valores de $\mathrm{DL}_{50}$ para os biótipos de Bidens spp. resistentes (B1, B2, B3 e B5) e suscetíveis (B6, B7, B8 e B9) ao foransulfuron + iodosulfuron e imazapic + imazapyr e Fator de Resistência (FR) para os biótipos resistentes comparados com os biótipos suscetíveis.

\begin{tabular}{cccccc}
\hline \multirow{2}{*}{ Biótipos } & \multirow{2}{*}{$\mathrm{LL}_{50}$} & \multicolumn{5}{c}{ Fator de Resistência (FR) } \\
\cline { 3 - 6 } & & B1 $^{2}$ & B3 & B2 & B5 \\
\hline & & \multicolumn{1}{c}{ Foransulfuron + iodosulfuron } \\
\hline B1 & 263,0 & --- & --- & --- & --- \\
B3 & $>800,0$ & -- & --- & --- & --- \\
B6 & 56,0 & 4,70 & $>14,29$ & --- & --- \\
B8 & 46,0 & 5,72 & $>17,39$ & --- & --- \\
\hline & & & & Imazapic + imazapyr \\
\hline B2 & 81,0 & --- & --- & --- & --- \\
B5 & $>800,0$ & --- & --- & --- & --- \\
B7 & 55,0 & --- & --- & 1,48 & $>14,58$ \\
B9 & 44,0 & --- & --- & 1,84 & $>18,18$ \\
\hline
\end{tabular}

$1-\mathrm{FR}=\mathrm{DL}_{50}$ Resistente/ $\mathrm{DL}_{50}$ Suscetível;

2 - O Biótipo B1 foi comparado com os Biótipos B6 e B8.

Os valores de Fator de Resistência (FR) encontrados foram baixos (Tabela 1) quando comparados com outros herbicidas testados em Bidens spp resistentes a inibidores da ALS. Os fatores de resistência para o foransulfuron + iodosulfuron, foram maiores para o biótipo B3 do que para o biótipo $\mathrm{B} 1$, devido que a $\mathrm{DL}_{50}$ do biótipo B3 foi maior que $800 \%$ da dose usual. Para o imazapic + imazapyr, os fatores de resistência foram 10 vezes maiores para o biótipo B5 do que para o biótipo B2.

Para cloransulan, chlorimuron e imazethapyr os valores de FR, em Bidens spp. foram, 144000, 4413 e 348, respectivamente (Hernandez, 2004). Em Bidens pilosa, para os herbicidas chlorimuron, nicosulfuron, metsulfuron e imazethapyr os valores de FR foram, 41; 174; 57 e 57; respectivamente (Christoffoleti, 2002). Verificando a resistência múltipla em Euphorbia heterophylla os FR's foram superiores a 17 para o inibidor da ALS (imazethapyr) e 40 para o herbicida inibidor da PROTOX (fomesafen) (Trezzi et al., 2004). 
As diferenças entre os valores encontrados em Bidens spp. podem ser explicadas, devido ao fato que os biótipos testados serem de diferentes localidades, sua resistência deve ter evoluído de forma independente e ainda alguns biótipos podem estar sofrendo pressão de seleção há mais tempo. Como os herbicidas testados neste trabalho são novos, provavelmente eles não selecionaram biótipos resistentes, o que explica os baixos valores encontrados.

Os resultados demonstram que foransulfuron + iodosulfuron e imazapic + imazapyr não controlam os biótipos com histórico de resistência, apresentando resistência cruzada aos herbicidas inibidores da ALS, já que, os controles obtidos situam-se abaixo do aceitável e dos valores obtidos com os biótipos suscetíveis.

Uma das alternativas para o manejo e prevenção de plantas daninhas resistentes aos herbicidas é a associação formulada de herbicidas. Esta é baseada no fato de que os ingredientes ativos controlam eficientemente os dois biótipos da mesma espécie, sendo que o biótipo resistente a um dos herbicidas é controlado pelo outro ingrediente ativo da mistura (Powles \& Holtum, 1994; Gould, 1995). Porém, esta associação deve ser com dois ingredientes ativos de diferentes mecanismos de ação, o que não ocorre com as duas associações aqui utilizadas, porque ambas utilizam a associação do mesmo mecanismo de ação (inibidores da enzima ALS).

Portanto, deve-se utilizar produtos alternativos ao mecanismo dos herbicidas inibidores da enzima ALS, na cultura do milho, para o controle de Bidens spp, destacando-se o herbicida mesotrione (inibidor de caroteno) e o herbicida atrazine (inibidor do FS II) na dose de $1200 \mathrm{~g} \mathrm{ha}^{-1}$ no estádio de pós-emergência precoce promoveram um controle aceitável (Hernandez, 2004). Alem disso, deve-se utilizar práticas culturais como rotação de culturas, manejo integrado de plantas daninhas e rotação dos métodos de controle (Christoffoleti, 2004).

Conclui-se que embora a mistura de foransulfuron + iodosulfuron e imazapic + imazapyr sejam novos herbicidas para a cultura de milho, não solucionam o problema de resistência em Bidens spp. Tal fato deve ser alertado aos agricultores para que evitem prejuízo e para adotarem medidas de prevenção contra a seleção de outros biótipos resistentes.

\section{AGRADECIMENTOS}

Ao CNPq, CAPES e FAPERGS, pelo apoio ao trabalho.

\section{LITERATURA CITADA}


BIANCHI, M. A. Programa de difusão do manejo integrado de plantas daninhas em soja. In: REUNIÃO DE PESQUISA DE SOJA DA REGIÃO SUL, 23, Porto Alegre, RS. Ata e Resumos... Porto Alegre, 1996. 125p.

CHRISTOFFOLETI, P.J. Curvas de dose-resposta de biótipos resistente e suscetível de Bidens pilosa aos herbicidas inibidores da ALS. Scientia Agricola, Piracicaba, v.3, p513-519, 2002.

CHRISTOFFOLETI, P.J; LÓPEZ-OVEJERO, R.F.; CARVALHO, J.C. Aspectos da resistência de plantas daninhas a herbicidas. 2. Ed. Campinas: HRAC-BR, 2004. $100 \mathrm{p}$.

GOULD, F. Comparisons between resistance management strategies for insects and weeds. Weed Technology, v.9, n.4, p.830-839, 1995.

HEAP, I. The international survey of herbicide resistant weeds. Disponível em: <http://www.weedscience.com>, em Dezembro, 2005.

HERNANDES, G. C. Resistência de Bidens spp. aos herbicidas inibidores da acetolactato sintase: análise de populações com marcadores moleculares e estratégias para seu manejo. Porto Alegre: UFRGS, 2004. 109 f. Dissertação (Mestrado) - Programa de Pós-Graduação em Fitotecnia, Faculdade de Agronomia, Universidade Federal do Rio Grande do Sul, Porto Alegre, 2004.

KISSMANN, K.G.; GROTH, D. Plantas infestantes e nocivas - Tomo I. São Paulo: BASF, 1992, 797 p.

POWLES, S.; HOLTUM, J. Herbicide resistence in plants: biology and biochemistry. Boca Raton: CRC Press, 1994. 353p.

TREZZI, M.M.; FELIPPI, C.L.; MATTEI, D.; SILVA, H.L.; DEBASTIANI, C.; NUNES, A.L.; VIDAL, R.A. Multiple Resistance of Acetolactate Synthase and Protoporphyrinogen Oxidase Inhibitors in Euphorbia heterophylla Biotypes. Journal of Environmantal Science and Healt, Virginia, v.40, n.1, p.1-9, 2004.

TREZZI, M.M.; VIDAL, R.A. Herbicidas inibidores da ALS. In: VIDAL, R.A.; MEROTTO JR, A. Herbicidologia. Porto Alegre: Evangraf, 2001, p. 25-36. 